\title{
Endocrine autoimmunity in families with type 1 diabetes: frequent appearance of thyroid autoimmunity during late childhood and adolescence
}

\author{
E. Bonifacio • A. Mayr • A. Knopff • A.-G. Ziegler
}

Received: 3 July 2008 / Accepted: 9 October 2008 / Published online: 19 November 2008

(C) Springer-Verlag 2008

\begin{abstract}
Aims/hypothesis Thyroid autoimmunity clusters with other endocrine and non-endocrine forms of autoimmunity. The aim of this study was to determine the chronological appearance of thyroid autoantibodies in relation to other forms of autoimmunity in at-risk children.

Methods The BABYDIAB study follows children of parents with type 1 diabetes. Children born in Germany between 1989 and 2000 were recruited at birth and followed up at 9 months and at 2, 5, 8, 11, 14 and 17 years. Antibodies to thyroid peroxidase were measured in samples taken at the last study visit in 1,489 children and in all previous samples in children who tested positive. Islet antibodies and antibodies to 21-hydroxylase and transglutaminase were also measured in all children. Median follow-up was 8 years.

Results The cumulative risk for developing antibodies to thyroid peroxidase was $20.3 \%$ (95\% CI $12.3-28.3)$ by age
\end{abstract}

\section{E. Bonifacio}

DFG Research Center and Cluster of Excellence for Regenerative

Therapies Dresden, Dresden University of Technology,

Dresden, Germany

\section{A. Mayr · A. Knopff $\cdot$ A.-G. Ziegler}

Forschergruppe Diabetes, Klinikum rechts der Isar,

Technische Universität München,

Munich, Germany

\section{A.-G. Ziegler}

Department of Endocrinology, Klinikum Schwabing,

Städt. Klinikum München,

Munich, Germany

\section{A.-G. Ziegler $(\bowtie)$}

Diabetes Research Institute,

Kölner Platz 1,

D-80804 Munich, Germany

e-mail: anziegler@1rz.uni-muenchen.de
14 years. The risk was increased in girls (adjusted HR 2.0; 95\% CI 1.2-3.4; $p=0.008$ ), in children who had multiple first-degree family history of type 1 diabetes (adjusted HR 3.3; 95\% CI 1.4-8.0; $p=0.006$ ) and in children who also had antibodies to GAD (adjusted HR 3.0; 95\% CI 1.5-5.9; $p=0.001$ ). Thyroid peroxidase antibody appearance was most common from age 8 years and was often the last autoantibody to develop in children with other autoantibodies.

Conclusions/interpretation Among children of patients with type 1 diabetes, the appearance of thyroid autoantibodies is frequent, is not synchronous to the appearance of other autoantibodies and is most common in late childhood and adolescence.

Keywords Islet autoantibodies - Thyroid autoimmunity . Type 1 diabetes

$\begin{array}{ll}\text { Abbreviations } \\ \text { GAD-Abs } & \text { GAD antibodies } \\ \text { IAA } & \text { insulin autoantibodies } \\ \text { IA-2-Abs } & \text { islet antigen } 2 \text { antibodies } \\ \text { 21-OH-Abs } & \text { 21-hydroxylase antibodies } \\ \text { TBST } & \text { tris buffered saline with Tween 20 } \\ \text { TPO-Abs } & \text { antibodies to thyroid peroxidase } \\ \text { tTG-Abs } & \text { antibodies to tissue transglutaminase }\end{array}$

\section{Introduction}

Autoimmunity to endocrine cells is a pathogenetic feature of diseases such as type 1 diabetes, Graves' disease, Hashimoto's thyroiditis and Addison's disease [1-4]. There is an association between endocrine autoimmune diseases [5-8], further suggesting shared pathogenetic mechanisms. 
A typical feature of such disorders is the appearance of circulating autoantibodies to endocrine cell proteins prior to clinical onset of disease [9]. The detection of autoantibodies denotes that a sensitisation to the target cell has occurred and autoantibody-positive individuals have an increased risk of developing clinical disease [10].

The appearance of the autoantibodies is often the first detectable sign of autoimmune pathogenesis and can be used to track the natural history during the preclinical period. Indeed, using cohorts of at-risk individuals studied from birth, it has been demonstrated for type 1 diabetes that autoantibodies to beta cell antigens such as insulin and GAD65 can appear already in the first year of life [11, 12] and that autoantibody appearance is determined by several susceptibility genes [13-15] and modified by environmental factors $[16,17]$. Similar studies have been performed for coeliac disease [8, 18]. Although thyroid autoantibodies have been identified long before antibodies associated with diabetes or with coeliac disease [19], the natural history of their development from birth is unknown. Here, we took advantage of the association of thyroid autoimmunity with type 1 diabetes [4] and studied the natural history of autoantibodies to thyroid peroxidase in participants in the BABYDIAB study [11], who were all children of parents with type 1 diabetes. Our results show that thyroid autoimmunity is common in children who are genetically at risk of contracting type 1 diabetes and that autoantibodies to thyroid peroxidase commonly appear in late childhood and adolescence. Screening at-risk populations from childhood may, therefore, be warranted for correction of thyroid function.

\section{Methods}

The BABYDIAB study examined the natural history of autoimmunity to islet antigens from birth in children of parents with type 1 diabetes [11]. Families were eligible to participate if one or both parents had type 1 diabetes. Recruitment into the study began in 1989 and ended in 2000. Recruitment was facilitated through advertisements in paediatric and patient journals, and in paediatric and neonatal clinics. Participation was voluntary. The study was coordinated by the Diabetes Research Institute in Munich through direct contact with the families and the family paediatrician. Cord blood was obtained in obstetric departments from eligible families who had consented to participation. Venous blood samples from the child during follow-up were obtained at paediatric clinics at age 9 months and at 2, 5, 8, 11, 14 and 17 years. The median follow-up time from birth to last sample was 8 years (range $0.75-18.5$ years). Questionnaires were completed at birth and at each paediatric visit. The cumulative dropout rate was $16.0 \%$ by age 5 years and $20.9 \%$ by age 8 years. All families gave written informed consent to participate in the BABYDIAB study. The study was approved by the Ethics Committee of Bavaria, Germany (Bayerische Landesärztekammer [Bavarian Medical Council] no. 95357).

For the present study, all children who were followed until at least 2 years of age were included $(n=1,489)$. Islet autoantibodies and antibodies to tissue transglutaminase $\mathrm{C}$ (tTG-Abs) were measured in samples taken at all scheduled visits. Antibodies to thyroid peroxidase (TPO-Abs) were measured in the last available blood sample from all these children. In 1,474 children there was also sufficient serum for measurement of antibodies to 21-hydroxylase (21-OH$\mathrm{Abs}$ ) in the last sample. In children found positive for TPOAbs or 21-OH-Abs, all earlier samples were also measured to determine the age of appearance of these antibodies. Children who developed type 1 diabetes $(n=36)$ were not followed after diagnosis.

Autoantibody measurements TPO-Abs were measured by a direct radiobinding assay according to the manufacturer's instructions (CentAK anti-TPO; Medipan, Dahlewitz/Berlin, Germany). Briefly, $50 \mu 1$ serum was incubated with ${ }^{125}$ I-labelled recombinant human TPO in assay buffer with $50 \mu \mathrm{l}$ protein A suspension for $30 \mathrm{~min}$. Subsequently $1 \mathrm{ml}$ assay buffer was added. Pellets were washed and counted in a gamma counter (Packard, Meridan, CT, USA). TPO-Abs were expressed as U/ml. Samples were defined as TPOAbs-positive above a threshold of $50 \mathrm{U} / \mathrm{ml}$ as suggested by the manufacturer and confirmed using QQ-plot analysis, which plots the distribution of the variable (autoantibody) against the autoantibody concentration, allowing assessment of where distribution deviates from normal [11]. The inter-assay coefficient of variation was $11 \%$ at $660 \mathrm{U} / \mathrm{ml}$ and $23 \%$ at $34 \mathrm{U} / \mathrm{ml}$.

21-OH-Abs were measured by radiobinding assay similar to those previously described for glutamic acid decarboxylase antibodies (GAD-Abs) and islet antigen 2 antibodies (IA-2-Abs) [10]. Briefly, $2 \mu 1$ serum was incubated with $20,000 \mathrm{cpm}$ of $\left[{ }^{35} \mathrm{~S}\right]$ methionine-labelled, in vitro-transcribed/translated recombinant human $21-\mathrm{OH}$ in $50 \mathrm{mmol} / 1$ Tris buffered saline containing 1\% Tween 20 (TBST). Antibody-bound 21-OH was recovered with protein-A sepharose beads (GE Healthcare Life Sciences, Amersham, UK), beads washed five times in TBST and scintillation counted in a Top Count Microplate Scintillation Counter; Packard). Relative antibody concentration was expressed as an index defined as: (cpm in the unknown sample - $\mathrm{cpm}$ in the negative standard)/(cpm in the positive standard $-\mathrm{cpm}$ in the negative standard $) \times 100$. The threshold was determined using QQ plots and was set at 6 index units, which also corresponded to the 99th percentile of 100 control children (mean age 7.5 years; interquartile range 
5.1-9.1). The inter-assay coefficient of variation was $16 \%$ at 41 index units.

IgA antibodies to tissue transglutaminase (tTG-Abs) were measured by ELISA according to the manufacturer's instructions (Eurospital, Trieste, Italy) and by radiobinding assay with $\left[{ }^{35} \mathrm{~S}\right]$ methionine-labelled, in vitro-transcribed/ translated recombinant human tTG as previously described [8]. Thresholds for positivity were determined using QQ plots and corresponded to the 95th percentile of control children without diabetes or coeliac disease for the ELISA and the 99th percentile of control samples for the radiobinding assay. Samples were defined as positive if they were detected above these thresholds in both assays.

Insulin autoantibodies (IAA), GAD autoantibodies (GAD-Abs) and IA-2 autoantibodies (IA2-Abs) were measured by radiobinding assays, as previously described $[11,20]$. The upper limits of normal were determined using Q-Q plots and corresponded to the 99th percentile of control children, i.e. 1.5 local units/ml for IAA, $25 \mathrm{WHO}$ units $/ \mathrm{ml}$ for GAD-Abs and $4 \mathrm{WHO}$ units/ml for IA2-Abs. Using these thresholds for positivity, the assays had sensitivities and specificities of 70 and $99 \%$ (IAA), 86 and $93 \%$ (GAD-Abs), 72 and 100\% (IA2-Abs) and 84 and $100 \%$ for multiple islet autoantibodies in the Third Diabetes Autoantibodies Standardization Program Proficiency Workshop [21]. The inter-assay coefficient of variation for samples with low autoantibody titre was $11 \%$ for IAA, $18 \%$ for GAD-Abs and $16 \%$ for IA2-Abs.

All antibody measurements were performed on coded samples that were blinded to the operator.

$H L A$ genotyping HLA DR and $D Q$ genotypes were determined in 1,413 children of parents with type 1 diabetes. The remaining 76 children did not provide a suitable sample for HLA typing. HLA-DRB1, HLA-DQA1 and HLA$D Q B 1$ alleles were typed using PCR-amplified DNA and non-radioactive sequence-specific oligonucleotide probes as described previously [13].

Notification of clinical disease Thyroid and other autoimmune disease status of the child and other family members was reported by response to questionnaires received at each study visit. None of the children were reported to have congenital hypothyroidism. In addition, as previously described [8], children who were persistently positive for tTG-Abs were recommended to have intestinal biopsies for diagnosis of coeliac disease. Families were not notified of thyroid autoantibody status and no recommendation with respect to thyroid function was provided to families of children positive for TPO-Abs.

Statistical analysis Time-to-event methods were used to calculate risks (life table analysis) and to compare autoan- tibody outcome for participants with different covariate categories (life table analysis and Cox proportional hazards model). In children with a positive autoantibody outcome, the age at the first positive sample was used as the event time. Analysis considered censoring in losses to follow-up and in participants with antibody-negative status at the follow-up visit age of their last autoantibody-negative sample. The log rank test was used for comparisons of covariate categories in life table analysis. HRs were calculated using Cox proportional hazards model. Variables that were significant $(p<0.05)$ in the univariate model were tested in the multivariate model using forward conditional analysis. The proportional hazards assumption in the Cox model was tested by examining the $\log$ minus $\log$ plot of each covariate for parallel curves and by using a timedependent Cox regression that included the covariate in question and the interaction between time and the covariate. The interaction was not significant for all covariates indicating that the hazards were proportional.

TPO antibody incidence was determined by calculating the incremental increase in risk at 2, 5, 8, 11 and 14 years corrected for the time interval between visits, and expressed as cases per 100 participants per year. For all analyses, a twotailed $p$ value of 0.05 was considered significant. All statistical analyses were performed using the Statistical Package for Social Science (SPSS 15.0; SPSS, Chicago, IL, USA).

\section{Results}

TPO antibody prevalence and time of appearance Screening for TPO-Abs in the last available sample of BABYDIAB children identified 63 children with TPO-Abs (Fig. 1a). Earlier samples from all positive children were subsequently measured to find the age of appearance of TPO-Abs. Five children had developed TPO-Abs at age 2 years, five at age 5 years, 32 at age 8 years and 21 at age 11 years or older. TPO-Abs were persistent in 41 children and transient in only one (the remainder did not have a later sample in order to assess persistency), quickly increasing to high titre in the majority of cases (data not shown).

The cumulative risk of developing TPO-Abs was $4.3 \%$ (95\% CI 3.0-5.6) by 8 years, $7.3 \%$ (95\% CI $5.1-9.5$ ) by 11 years and $20.3 \%$ (95\% CI 12.3-28.3) by 14 years (Fig. 1 b). The incidence of new cases was 0.15 per 100 children/year at ages 2 and 5 years, rising to 1 per 100/year at 8 and 11 years and 4 per 100/year at age 14 years. Of the 63 antibody-positive children, eight had reported clinical hypothyroidism 1.5 to 7 years after the first detection of TPO-Abs and 52 had no reported clinical thyroid disease (three participants did not complete questionnaires). Hypothyroidism was reported in two children who were TPO-Abs negative. 
a

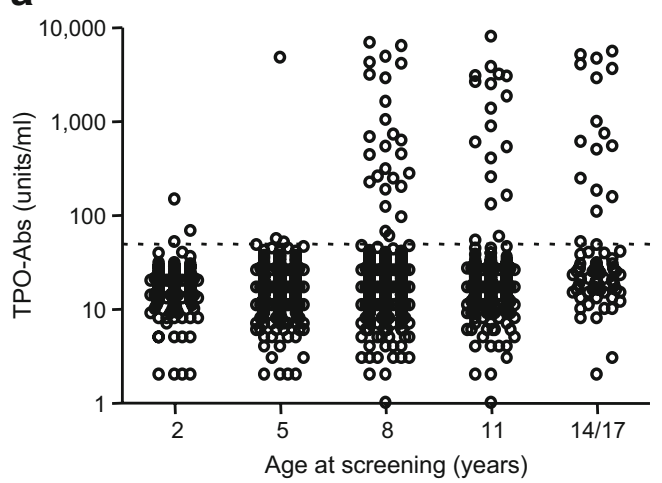

b

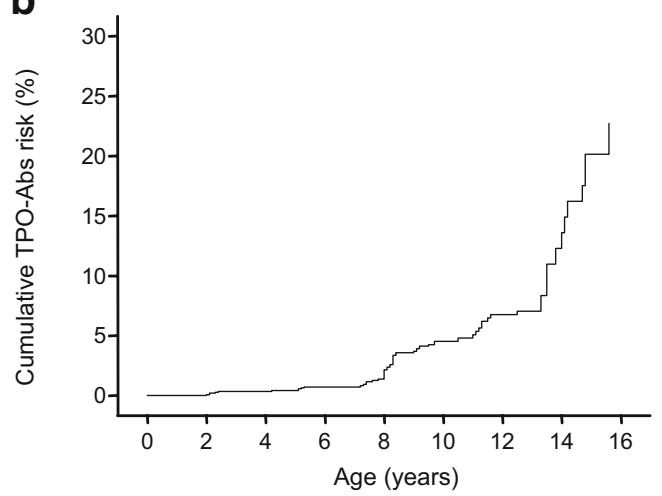

Fig. 1 Appearance of TPO-Abs in participating children of parents with type 1 diabetes in the BABYDIAB study. a TPO-Abs titre in the last available sample from 1,489 children in the BABYDIAB cohort. Samples are shown for the visits at $2(n=148), 5(n=435), 8(n=553)$, $11(n=279)$ and $14 / 17(n=74)$ years. b Cumulative life table risk of developing TPO-Abs in the BABYDIAB children. Numbers of children remaining negative for TPO-Abs on follow-up with respect to age: baseline ( 0 years) $(n=1489), 2$ years $(n=1489), 5$ years $(n=1340), 8$ years $(n=901), 11$ years $(n=342), 14$ years $(n=71)$

Factors affecting the risk of TPO development The risk of developing TPO-Abs was increased in girls (HR 1.8; 95\% CI $1.1-3.1 ; p=0.02)$, in children who were islet autoantibody-positive (HR 2.5; 95\% CI 1.3-4.7; $p=0.005$ ), in children who had more than one family member affected with type 1 diabetes (HR 4.1; 95\% CI $1.8-9.5 ; p=0.001$ ) and in children with the HLA DRB1*03/DRB1*04$D Q B 1 * 0302$ genotype (HR 2.2; 95\% CI $1.2-4.3 ; p=0.02$ ) (Table 1). With respect to islet autoantibodies, the risk of developing TPO-Abs was associated with the presence of GAD-Abs (HR 3.1; 95\% CI 1.6-6.0; $p=0.0006$ ), whereas IAA and/or IA-2-Abs in the absence of GAD-Abs were not associated with TPO-Abs risk (HR 0.8). TPO-Abs risk was also increased in children who developed type 1 diabetes (HR $4.4 ; p=0.01$ ).

Female sex (adjusted HR 2.0; 95\% CI 1.2-3.4; $p=$ 0.008 ), positivity for GAD-Abs (adjusted HR 3.0; 95\% CI $1.5-5.9 ; p=0.001)$ and multiple first-degree family history of type 1 diabetes (adjusted HR 3.3; 95\% CI 1.4-8.0; $p=$ 0.006 ) contributed to the risk of developing TPO-Abs in the Cox proportional hazards model. Inclusion of $D R B 1 * 03$ / $D R B 1 * 04-D Q B 1 * 0302$ genotype or development of type 1 diabetes did not further improve the model $(p=0.09$ and $p=0.5$, respectively).

Relationships between TPO-Abs and other autoantibodies Within the same cohort, 110 children developed islet autoantibodies (IAA, GAD-Abs and/or IA2-Abs), 66 developed tTG-Abs and seven developed 21-OH-Abs (Fig. 2a). Overlap between antibodies was observed, but the vast majority of positive cases did not have autoantibodies to multiple organs. TPO-Abs developed in $12(11 \%)$ children with islet autoantibodies, six $(9 \%)$ children with tTG-Abs and two (29\%) children with 21-OH-Abs. Five of the 36 children who progressed to type 1 diabetes developed TPO-Abs $(n=3)$ and/or tTG-Abs $(n=3)$ prior to diabetes onset; none developed 21-OH-Abs. The age of appearance of each antibody differed, with islet autoantibodies generally preceding tTG-Abs and TPO-Abs being the last to develop (Fig. 2b). In the seven children positive for 21-OH-Abs, antibodies developed from age 2 years until 11 years (cumulative prevalence $1.7 \%$ by age 11 years). In 13 of 18 children in whom TPO-Abs plus another autoantibody developed, TPO-Abs were the last to appear, in two others TPO-Abs preceded islet autoantibodies and tTG-Abs, respectively. Only in three children did TPO-Abs occur at the same age as islet autoantibodies $(n=2)$ or 21-OH-Abs $(n=1)$ (Fig. 2c). Notably, six of nine children who reported clinical Hashimoto's disease had islet autoantibodies $(n=3)$, tTG-Abs $(n=3)$ or 21-OH-Abs $(n=1)$.

Overall, 224 children developed at least one autoantibody (islet Abs, TPO-Abs, tTG-Abs, 21-OH-Abs). The cumulative risk for any autoantibody was $21.5 \%$ by 11 years (95\% CI $18.5-24.5 \%$ ) and $32 \%$ by 14 years $(24-40 \%)$ (Fig. 3a). Remarkably, selection of children with the $H L A$ $D R 3 / 4, H L A-D R 4 / 4$ or $H L A-D R 3 / 3$ genotypes, which are associated with type 1 diabetes and coeliac disease, identified 199 children who had a $46 \%$ risk $(95 \%$ CI 34 58) of developing islet-Abs, tTG-Abs or TPO-Abs by age 11 years (Fig. 3b).

\section{Discussion}

Thyroid autoimmune disease is preceded by autoantibodies to TPO and thyroglobulin, and is often associated with autoimmune disorders such as type 1 diabetes [14, 22]. Here we show that TPO-Abs are frequent in children at risk of developing type 1 diabetes, appearing in late childhood and after the peak incidence of islet autoantibodies. 
Table 1 Factors affecting TPO-Abs risk (univariate analysis)

\begin{tabular}{|c|c|c|c|c|}
\hline Factor & Participants $(n)$ & TPO-positive (n) & $\operatorname{HR}(95 \% \mathrm{CI})$ & $p$ value \\
\hline \multicolumn{5}{|l|}{$\overline{\operatorname{Sex}}$} \\
\hline Girls & 727 & 40 & $1.8(1.1-3.1)$ & \multirow[t]{2}{*}{0.02} \\
\hline Boys & 762 & 23 & Reference & \\
\hline \multicolumn{5}{|c|}{ Type 1 diabetes family history } \\
\hline One parent only & 1451 & 57 & Reference & \multirow[b]{2}{*}{0.001} \\
\hline Multiple & 38 & 6 & $4.1(1.9-9.5)$ & \\
\hline \multicolumn{5}{|l|}{ HLA } \\
\hline DR $3 / 4$ & 141 & 11 & $2.20(1.15-4.2)$ & \multirow[t]{2}{*}{0.02} \\
\hline Non-DR 3/4 & 1,272 & 50 & Reference & \\
\hline \multicolumn{5}{|c|}{ Islet autoantibodies } \\
\hline Positive & 110 & 12 & $2.27(1.2-4.3)$ & \multirow[t]{2}{*}{0.01} \\
\hline Negative & 1,379 & 51 & Reference & \\
\hline \multicolumn{5}{|l|}{ GAD-Abs } \\
\hline Positive & 86 & 11 & $2.69(1.4-5.3)$ & \multirow[t]{2}{*}{0.003} \\
\hline Negative & 1,403 & 52 & Reference & \\
\hline \multicolumn{5}{|l|}{ tTG-Abs } \\
\hline Positive & 66 & 6 & $2.29(1.0-5.35)$ & \multirow[t]{2}{*}{0.05} \\
\hline Negative & 1,423 & 57 & Reference & \\
\hline \multicolumn{5}{|l|}{ Type 1 diabetes } \\
\hline Positive & 30 & 3 & $4.4(1.4-14.2)$ & \multirow[t]{2}{*}{0.01} \\
\hline Negative & 1,459 & 60 & Reference & \\
\hline
\end{tabular}

The unique aspect of this study is that it is prospective from birth, thereby allowing us to determine the age of autoantibody appearance in each participant. TPO autoantibodies were measured by a sensitive radiobinding assay. Moreover, because multiple samples were available for the children, positivity could be confirmed in most cases. Additionally, all children had been extensively studied for the development of autoantibodies associated with type 1 diabetes [11], coeliac disease [8] and Addison's disease.

One limitation of the present study is that it is not population-based, but was performed in a cohort enriched for type 1 diabetes susceptibility. We are therefore unable to relate our findings to children in the general population and unable to provide a background frequency of the antibodies measured. However, to its advantage and unlike other similar cohorts [14], the BABYDIAB cohort has not been further selected on the basis of HLA genotype. A second limitation is that whereas the majority of participants have been followed through to late childhood, only a minority have been followed to adolescence and therefore TPO-Abs incidences reported for adolescence are imprecise. Moreover, it is possible that a number of children who developed TPOAbs in adolescence will subsequently become islet autoantibody-positive. Similarly, since children who developed a

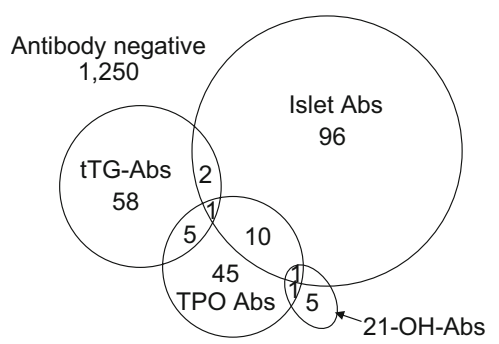

b

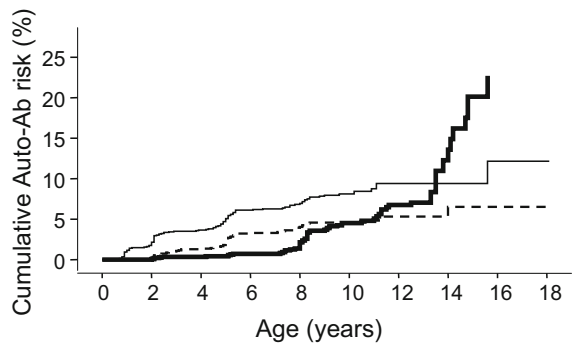

C

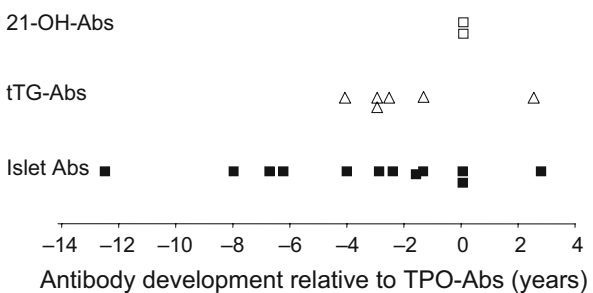

Fig. 2 TPO-Abs in relation to the appearance of other autoantibodies. a Venn diagram showing numbers of BABYDIAB children with one or more of the following antibodies: TPO-Abs, tTG-Abs, islet Abs (insulin, GAD or IA-2) and 21-OH-Abs. b Cumulative life table risk for development of autoantibodies in the BABYDIAB children. Three different outcomes are shown in the 1,489 children: bold line, cumulative frequency of TPO-Abs; thin line, islet autoantibodies; and dashed line, transglutaminase antibodies. c Time relationship of autoantibody appearance in children who had antibodies to more than one organ. The age of appearance of islet antibodies (black squares), transglutaminase antibodies (triangles) and 21-hydroxylase antibodies (white squares) relative to the appearance of TPO antibodies is shown for the 18 children who had TPO antibodies plus at least one other autoantibody 

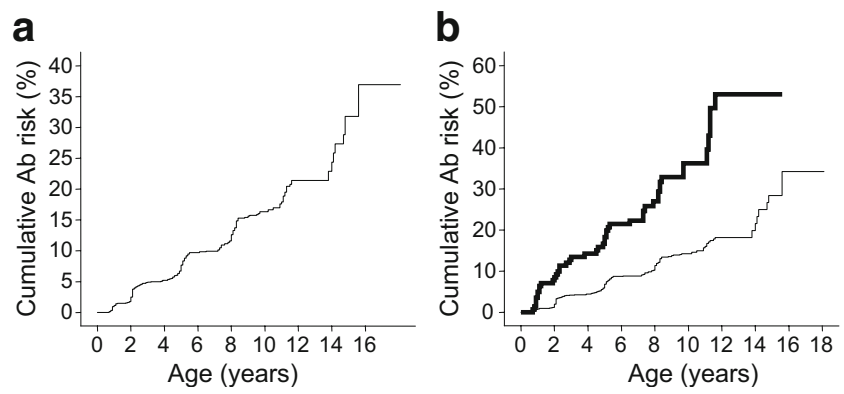

Fig. 3 Combined autoantibody development. a Cumulative life table risk for development of any autoantibody (TPO-Abs, islet Abs, tTGAbs, 21-OH-Abs) in the BABYDIAB children. Numbers of children remaining negative for TPO-Abs on follow-up with respect to age: baseline $(0$ years $)(n=1489), 2$ years $(n=1461), 5$ years $(n=1270)$, 8 years $(n=805), 11$ years $(n=265), 14$ years $(n=53)$. b Cumulative life table risk for development of any autoantibody in children after stratification for $H L A-D R / D Q$ genotype as high risk (HLA-DR3,4$D Q 8$; $D R 4-D Q 8, D R 4-D Q 8 ; D R 3,3$; thick line) or moderate/low risk (all other genotypes; thin line). Risk between the groups was significantly different $\left(p=7 \times 10^{-9}\right)$. Numbers of children remaining negative for TPO-Abs on follow-up with respect to age: high-risk HLA group: baseline ( 0 years) $(n=141), 2$ years $(n=131), 5$ years $(n=108)$, 8 years $(n=66), 11$ years $(n=20), 14$ years $(n=3)$; moderate/low-risk HLA group: baseline ( 0 years) $(n=1267), 2$ years $(n=1254)$, 5 years $(n=1112), 8$ years $(n=713), 11$ years $(n=240), 14$ years $(n=48)$

type 1 diabetes were no longer followed after disease onset, we cannot comment on the development of TPOAbs or 21-OH-Abs after diabetes onset, possibly leading to bias in the observed antibody prevalence in this minority subgroup. By the same token, numbers of positive participants become small when subgroups are analysed, thereby limiting precision of risk estimates. A third limitation is that, because of the study design, which included screening for positive cases in the last available sample, we may have missed transient appearance of thyroid or adrenal autoimmunity during early childhood. However, the screening strategy does not affect the timing of the first appearance of persistent TPO-Abs or 21-OH Abs, since all previous samples were measured in children who were persistently positive. It should be noted that islet and tTG-Abs were measured in all samples, which could have affected the relative frequencies of these antibodies in comparison to TPO-Abs and 21-OH antibodies. Fourth, and importantly, there was no clinical follow-up of participants for thyroid disease, thereby limiting interpretation of the TPO-Abs findings with respect to clinical disease risk. Finally, the findings for all antibodies are dependent upon the thresholds for detection, and the sensitivity and specificity of the assays used. We used assays that on current knowledge have high specificity and sensitivity. We acknowledge, however, that antibody frequencies are likely to differ, if other assays and/or antibody classification strategies are used.
Previous studies have shown that autoimmune thyroid disease is frequent in females and that thyroid autoantibodies occur in $8 \%$ to $50 \%$ of patients with type 1 diabetes [23-31] and in $8 \%$ to $25 \%$ of their relatives [2, 6]. Our findings are consistent with these in that TPO-Abs were more frequent in girls than boys and were very frequent in this at-risk type 1 diabetes cohort. Remarkably, the frequency of TPO-Abs was already $7.3 \%$ by age 11 years and reached $20 \%$ by age 14 years. The high frequency of TPO-Abs in our study may be due to the use of a more sensitive assay than used in previous studies. However, the vast majority of positive children had TPO-Abs titres well beyond the threshold for positivity, and the frequency of TPO-Abs detected in the 2 and 5 year screening samples was less than $1 \%(5 / 583)$, indicating that the assay was highly specific. Similarly to results reported in patients with type 1 diabetes, the frequency of 21-OH Abs was around 1 to $2 \%$ in our cohort $[5,32]$.

The major novel finding of this study was the age of appearance of TPO-Abs and their appearance relative to autoantibodies associated with diabetes and coeliac disease. Unlike insulin and GAD-Abs that have an early peak incidence at around 1 to 2 years of age in this cohort [33], TPO-Abs were rare until after 5 years of age. TPO-Abs were associated with GAD-Abs, but were not associated with other islet autoantibodies in the absence of GAD-Abs. The association with GAD-Abs is consistent with previous findings in participants with so-called polyendocrine autoimmunity [34], which is also more frequent in females [1]. In the majority of children with both GAD-Abs and TPOAbs, GAD-Abs appeared before TPO-Abs. Similarly, TPOAbs appeared after tTG-Abs in most children in whom both antibodies occurred. Thus, the onset of autoimmunity is not synchronised for all target organs, and for each autoimmune target there appear to be distinct periods during growth that are associated with augmented risk for autoimmunity to develop. This seems to refute theories of a common antigenic basis or common environmental cause. Nevertheless, the very high frequency of autoimmunity of any type in these at-risk type 1 diabetes children suggests a common genetic susceptibility to autoimmunity that is partially, but not only HLA-based $[35,36]$.

The findings of this study are potentially relevant to screening and prevention of autoimmune disease. First, on the basis of HLA genotype and a first-degree family history of type 1 diabetes, we were able to identify a subgroup of children who had an almost $50 \%$ risk of developing autoimmunity during childhood. Second, several of the children who developed TPO-Abs subsequently developed clinical autoimmune thyroid disease. We suggest, therefore, that screening for thyroid dysfunction may be warranted as early as from late childhood in selected relatives of patients with type 1 diabetes. 
Acknowledgements This study was supported by grants from the Juvenile Diabetes Research Foundation (JDRF no. 1-2006-665), the Foundation 'Das Zuckerkranke Kind' and the Deutsche Diabetesgesellschaft. We thank M. Hummel and C. Winkler for expert assistance, and all families and paediatricians in Germany who participated in the study.

Duality of interest The authors declare that there is no duality of interest associated with this manuscript.

\section{References}

1. Betterle C, Dal Pra C, Mantero F, Zanchetta R (2002) Autoimmune adrenal insufficiency and autoimmune polyendocrine syndromes: autoantibodies, autoantigens, and their applicability in diagnosis and disease prediction. Endocrine Rev 23:327-364

2. Jaeger C, Hatziagelaki E, Petzoldt R, Bretzel RG (2001) Comparative analysis of organ-specific autoantibodies and celiac disease-associated antibodies in type 1 diabetic patient, their firstdegree relatives, and healthy control subjects. Diabetes Care 24:27-32

3. Hummel M, Bonifacio E, Stern M, Dittler J, Schimmel A, Ziegler AG (2000) Development of celiac disease-associated antibodies in offspring of parents with Type 1 diabetes. Diabetologia 43:10051011

4. Kordonouri O, Klinghammer A, Lang EB, Grüters-Kieslich A, Grabert M, Holl RW (2002) Thyroid autoimmunity in children and adolescents with type 1 diabetes. Diabetes Care 25:13461350

5. Barker JM, Yu J, Yu L et al (2005) Autoantibody "subspecificity" in type 1 diabetes. Diabetes Care 28:850-855

6. Hanukoglu A, Mizrachi A, Dalal I et al (2003) Extrapancreatic autoimmune manifestations in type 1 diabetes patients and their first-degree relatives. Diabetes Care 26:1235-1240

7. Betterle C, Zanette F, Pedini B et al (1984) Clinical and subclinical organ-specific autoimmune manifestations in Type 1 (insulin-dependent) diabetic patients and their first-degree relatives. Diabetologia 26:431-436

8. Hummel S, Hummel M, Banholzer J et al (2007) Development of autoimmunity to transglutaminase $\mathrm{C}$ in children of patients with type 1 diabetes: relationship to islet autoantibodies and infant feeding. Diabetologia 50:390-394

9. Liu E, Eisenbarth GS (2007) Accepting clocks that tell time poorly: fluid-phase versus standard ELISA autoantibody assays. Clin Immunol 125:120-126

10. Scofield RH (2004) Autoantibodies as predictors of disease. Lancet 386:1544-1546

11. Ziegler AG, Hummel M, Schenker M, Bonifacio E (1999) Autoantibody appearance and risk for development of childhood diabetes in offspring of parents with type 1 diabetes: the 2-year analysis of the German BABYDIAB Study. Diabetes 48:460 468

12. Achenbach P, Bonifacio E, Koczwara K, Ziegler AG (2005) Natural history of type 1 diabetes. Diabetes 54(Suppl. 2):S25-S31

13. Walter M, Albert E, Conrad M et al (2003) IDDM2/insulin VNTR modifies risk conferred by IDDM1/HLA for development of Type 1 diabetes and associated autoimmunity. Diabetologia 46:712-720

14. Barker JM, Barriga KJ, Yu L et al (2004) Diabetes Autoimmunity Study in the Young. Prediction of autoantibody positivity and progression to type 1 diabetes: Diabetes Autoimmunity Study in the Young (DAISY). J Clin Endocrinol Metab 89:3896-3902

15. Knip M, Kukko M, Kulmala P et al (2002) Humoral beta-cell autoimmunity in relation to HLA-defined disease susceptibility in preclinical and clinical type 1 diabetes. Am J Med Genet 115:4854

16. Ziegler AG, Schmid S, Huber D, Hummel M, Bonifacio E (2003) Early infant feeding and risk of developing type 1 diabetesassociated autoantibodies. JAMA 290:1721-1728

17. Norris JM, Barriga K, Klingensmith G et al (2003) Timing of cereal exposure in infancy affects risk of islet autoimmunity. The Diabetes Autoimmunity Study in the Young. JAMA 290:17131720

18. Norris JM, Barriga K, Hoffenberg EJ et al (2005) Risk of celiac disease autoimmunity and timing of gluten introduction in the diet of infants at increased risk of disease. JAMA 293:2343-2351

19. Czarnocka B, Ruf J, Ferrand M, Carayon P, Lissitzky S (1985) Purification of the human thyroid peroxidase and its identification as the microsomal antigen involved in autoimmune thyroid diseases. FEBS Lett 190:147-152

20. Naserke HE, Bonifacio E, Ziegler AG (1999) Immunoglobulin G insulin autoantibodies in BABYDIAB offspring appear postnatally: sensitive early detection using a protein A/G-based radiobinding assay. J Clin Endocrinol Metab 84:1239-1243

21. Torn C, Mueller PW, Schlosser M, Bonifacio E, Bingley PJ, and participating laboratories (2008) Diabetes Antibody Standardization Program: evaluation of assays for autoantibodies to glutamic acid decarboxylase and islet antigen-2. Diabetologia 51:846-852

22. Lam-Tse WK, Batstra MR, Koeleman BP et al (2003) The association between autoimmune thyroiditis, autoimmune gastritis and type 1 diabetes. Pediatr Endocrinol Rev 1:22-37

23. Riley WJ, Maclaren WJ, Lezotte DC, Spillar RP, Rosenbloom AL (1981) Thyroid autoimmunity in insulin-dependent diabetes mellitus: the case for routine screening. J Pediatr 99:350-354

24. McKenna MJ, Hershkowitz R, Wolfsdorf JI (1990) Screening for thyroid disease in children with IDDM. Diabetes Care 13:801803

25. Burek CL, Rose NR, Guire KE, Hoffman WH (1990) Thyroid autoantibodies in black and white children and adolescents with type 1 diabetes mellitus and their first degree relatives. Autoimmunity 7:157-167

26. Lorini R, d'Annunzio G, Vitali L, Scaramuzza A (1996) IDDM and autoimmune thyroid disease in the pediatric age group. J Pediatr Endocrinol Metab 9(Suppl. 1):89-94

27. Maugendre D, Massart C, Karacatsanis C et al (1997) Increased prevalence of thyroid autoantibodies and subclinical thyroid failure in relatives of patients with overt endocrine diseaseassociated diabetes but not type 1 diabetes alone. Diabetes Metab 23:302-307

28. Lindberg B, Ericsson UB, Ljung R, Ivarsson SA (1997) High prevalence of thyroid autoantibodies at diagnosis of insulindependent diabetes mellitus in Swedish children. J Lab Clin Med 130:585-589

29. Chang CC, Huang CN, Chuang LM (1998) Autoantibodies to thyroid peroxidase in patients with type 1 diabetes in Taiwan. Eur J Endocrinol 139:44-48

30. McCanlies E, O’Leary LA, Foley TP et al (1998) Hashimoto thyroiditis and insulin- dependent diabetes mellitus: differences among individuals with and without abnormal thyroid function. J Clin Endocrinol Metab 83:1548-1551

31. Roldan MB, Alonso M, Barrio R (1999) Thyroid autoimmunity in children and adolescents with type 1 diabetes mellitus. Diabetes Nutr Metab 12:27-31

32. Peterson P, Salmi H, Hyöty H et al (1997) Steroid 21-hydroxylase autoantibodies in insulin-dependent diabetes mellitus. Childhood Diabetes in Finland (DiMe) Study Group. Clin Immunol Immunopathol 82:37-42

33. Hummel M, Bonifacio E, Schmid S, Walter M, Knopff A, Ziegler AG (2003) Brief communication: early appearance of islet 
autoantibodies predicts childhood type 1 diabetes in offspring of diabetic parents. Ann Intern Med 140:882-886

34. Christie MR, Genovese S, Cassidy D et al (1994) Antibodies to islet $37 \mathrm{k}$ antigen, but not to glutamate decarboxylase, discriminate rapid progression to IDDM in endocrine autoimmunity. Diabetes 43:1254-1259
35. Levin L, Tomer Y (2003) The etiology of autoimmune diabetes and thyroiditis: evidence for common genetic susceptibility. Autoimmun Rev 2:377-386

36. Ide A, Eisenbarth GS (2003) Genetic susceptibility in type 1 diabetes and its associated autoimmune disorders. Rev Endocr Metab Disord 4:243-253 